\title{
Design Optimization of a Mecanum Wheel to Reduce Vertical Vibrations by the Consideration of Equivalent Stiffness
}

\author{
Jong-Jin Bae and Namcheol Kang \\ School of Mechanical Engineering, Kyungpook National University, 80 Daehakro, Bukgu, Daegu 41566, Republic of Korea \\ Correspondence should be addressed to Namcheol Kang; nckang@knu.ac.kr
}

Received 26 October 2015; Revised 5 February 2016; Accepted 14 February 2016

Academic Editor: Carlo Trigona

Copyright (C) 2016 J.-J. Bae and N. Kang. This is an open access article distributed under the Creative Commons Attribution License, which permits unrestricted use, distribution, and reproduction in any medium, provided the original work is properly cited.

\begin{abstract}
Mecanum wheels are capable of moving a vehicle to any direction instantaneously by the combination of independent wheel rotations. Because the mecanum wheel is composed of a hub and rollers, however, it has unavoidable drawbacks such as vertical and horizontal vibrations due to the sequential contacts between rollers and ground. In order to investigate the dynamic characteristics of a mecanum wheel, we made a prototype and performed experiments to measure the vertical vibrations. Interestingly, it was observed that the vertical accelerations were asymmetric with respect to the average value of signals; the vibration signals of upward and downward directions show quite different shape. This asymmetric phenomenon was confirmed through the dynamic simulations performed by RecurDyn. In addition, the peak-to-peak and RMS values of the displacements and accelerations were calculated to investigate the effects of the curvature of rollers on the vertical vibrations of the vehicle. Furthermore, we proposed a mecanum wheel having a spring to attenuate the vibrations. It was also noted that the significant reduction of the vertical accelerations was observed due to the absence of the spring. Finally, considering the equivalent stiffness of the mecanum wheel for several different fillet radii, we found the optimal geometric design which minimizes the vertical vibration of a mecanum wheel.
\end{abstract}

\section{Introduction}

Industrial and personal requirements for convenient maneuverability of vehicles have attracted much attention recently. However, the mechanisms of robots or vehicles with a conventional wheel are restricted to only one directional movement. For example, vehicles or robots equipped with classical steering system are difficult to move to the lateral direction and require the radius of gyration for turning. Therefore, those platforms are hard to rotate or avoid obstacles in narrow indoor spaces. For these reasons, a variety of wheels and driving platforms for omnidirectional movement have been developed to enhance the maneuverability of the vehicle or mobile robot. For example, the castor wheel and the special wheel type are used for the omnidirectional platform [1].

The castor wheel type is that the vehicle or the mobile robot is designed with the combination of the steered and conventional wheel [2-4]. However, the steered wheel has the high wheel scrubbing when it rotates with respect to its vertical axis for the change of the driving direction [5]. In the special wheels, passive rollers are oriented against the wheel shaft and connected to the wheel circumferentially. Also, a vehicle using special wheels is capable of omnidirectional movement by the combination of independent wheel rotations. Among the special wheels, the omniwheel, the passive roller axis being skew to the wheel axis at a $90^{\circ}$ angle, is generally used with single and double row types [6-8]. The vehicle with the omniwheel is usually composed of three or four wheels, but four-wheeled vehicle is generally preferred for the stability of the platform.

Another type is a mecanum wheel which is initially designed by Ilon [9]. The mecanum wheel has high load capacity, and it is usually used for a wheel chair or fork lift $[10,11]$. The configuration and advantage of mecanum wheel are as follows. The passive rollers are oriented at a $\pm 45^{\circ}$ degree wheel axis and are connected to wheel around [1215]. Typically the mecanum wheel consists of several passive rollers, forks, and a hub or disk. The hub, which is connected to the actuating motor, is a part to support the forks and rollers, and the passive rollers generate the driving force by the contact with the ground. Also, the vehicle is able to move 
to any direction by the combination of each wheel motion independently controlled.

In spite of the advantage such as the omnidirectional motion, the mecanum wheel has the unavoidable vibration due to its structural shape $[3,15,16]$. A vehicle with mecanum wheels can be excited vertically because the rollers transfer the impact forces to the vehicle body due to the consecutive contacts of rollers with ground. Although the contact between the mecanum wheel and ground itself is continuous, the contact points of the rollers are discontinuous. The discontinuity of the rollers causes the vertical vibration of a vehicle and a mobile robot. These unintended vibrations can reduce the positioning accuracy as well as the ride comfort of a wheel chair passenger.

Although the mecanum wheel has the well-known drawback of vibration problem, few studies have been conducted in prior research efforts to minimize the vibration. Nevertheless, in the other wheels to offer the omnidirectional movement, few studies have been conducted to reduce the vibration. For example, Byun proposed the alternative omniwheel of which outer roller is connected at each inner roller [17-19]. Unlike the classical omniwheel, this wheel has the continuous contact between the wheel and ground because the gaps between rollers are eliminated by the combination of inner and outer rollers. However, this approach is difficult to apply for the mecanum wheel, and the driving efficiency can be decreased by the friction between the inner and outer rollers. On the other hand, the ball wheel or spherical motor has been considered for the continuous contact between the wheel and ground [20-22]. The several ball wheels are installed under the vehicle body, and they can be rotated by the motion of the special or conventional wheels. This type also has the disadvantage such as the wear and tear of the ball. However, there remains a need to quantitatively interpret the vibration mechanism by using the experiment and dynamic simulation.

For these reasons, we focused on the quantitative analysis of the vertical vibration of the vehicle with built-in mecanum wheels. To do this, we designed the prototypes of the mecanum wheels and observed the asymmetric vertical acceleration of the wheel. In order to investigate the vertical vibration of the mecanum wheel, the dynamic simulation was performed using RecurDyn [23]. The parameter study was also performed to determine the optimal curvature range to minimize the vertical vibration. To additionally reduce the vertical vibration, we proposed the alternative design of the fork using the spring to attenuate the vibration. Finally, the equivalent stiffness of the fork was calculated using the finite element analysis.

\section{Experiment}

A mecanum wheel was designed, and multiple prototypes were implemented to investigate their dynamic characteristics. The proposed design of the mecanum wheel is consisted of a hub and eight forks and rollers for the wheel, as shown in Figure 1. The diameter and width of the mecanum wheel were designed to be $320 \mathrm{~mm}$ and $150 \mathrm{~mm}$, respectively. The hub and forks were made of aluminum, and the rollers were made

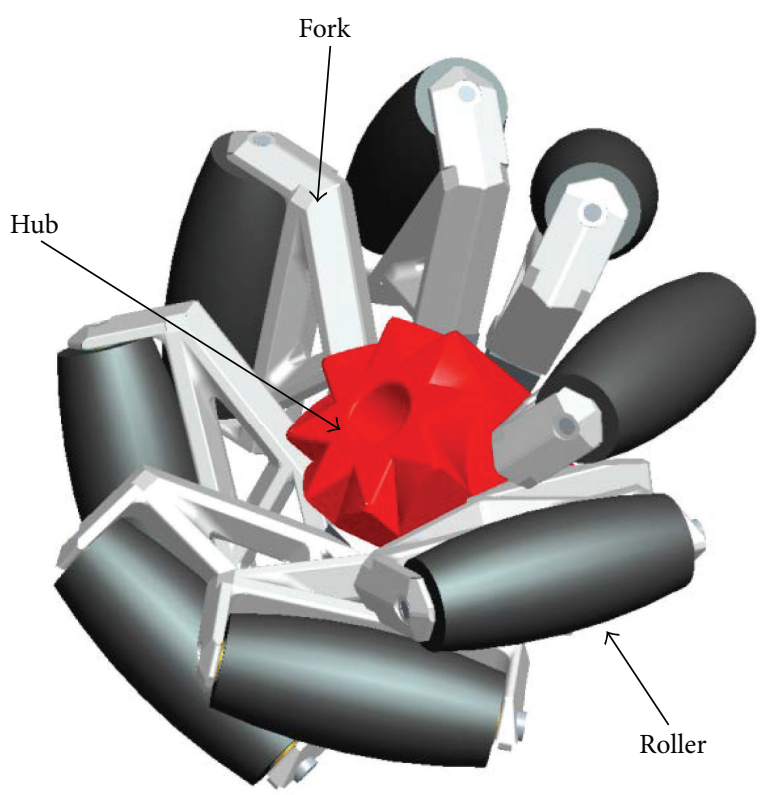

Figure 1: Schematic of the proposed mecanum wheel and its components.

of acrylic materials. The eight forks, which were inclined at angles of $45^{\circ}$ with respect to each other, were separately connected to the hub by two bolts for easy installation and replacement in the case of damage or malfunction. Moreover, eight rollers were installed through the bearings at each fork to rotate freely by the action of frictional forces generated upon contact with the ground.

Experiments were carried out to observe the vertical vibrations of the proposed mecanum wheel. As shown in Figure 2, the prototypes of the mecanum wheels are installed on a four-wheel driving wheelchair, with suspension on each wheel to improve ride quality. The vertical accelerations of the mecanum wheel were measured when the wheelchair executed translational motion along straight paths. In order to avoid the effects of the suspension, an accelerometer (Type 4507, Brüel \& Kjær) was installed underneath it (see Figure 2(b)). Additionally, the experimental measurements were performed on a flat and smooth ground surface to minimize the effects of road conditions. Low- and highspeed movements were applied to the wheelchair by manual operations in order to determine the effect of the speed dependency on the vertical vibration of the mecanum wheel. Time signals of the vertical acceleration were acquired at a $10 \mathrm{kHz}$ sampling frequency, as shown in Figure 3. As the eight rollers contacted the ground consecutively, contact forces were periodically generated. Although the time signals did not elicit a distinct time period due to the manual operation, maximum accelerations occurred as many times as the roller contacted the ground. Furthermore, it was observed that the magnitudes of the vertical accelerations at high speeds were much higher than those at low speeds, and the periods at high speeds were much shorter than those at low-speed movements.

Interestingly, it was also noted that the accelerations along the positive (upward) direction were much greater 


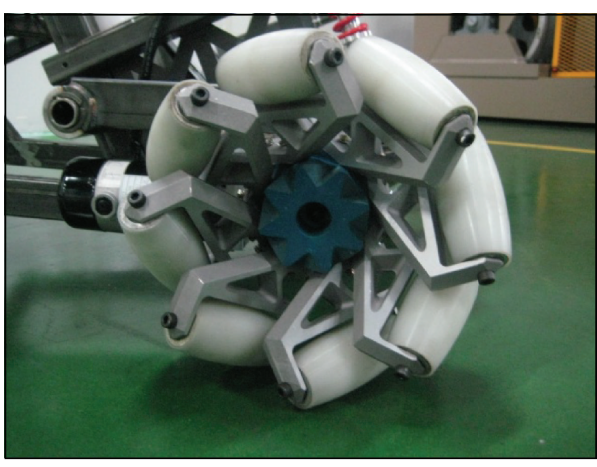

(a) Side view of the prototype

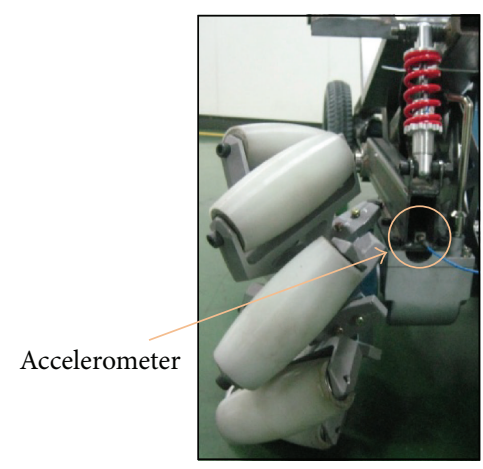

(b) Location of the accelerometer to measure the vertical vibration of the wheel

FIGURE 2: Prototype of the proposed mecanum wheel and sensor location for the measurement of vertical vibrations.

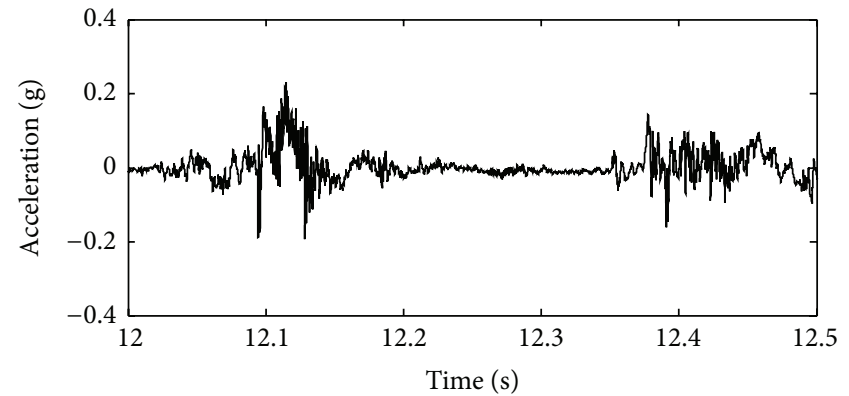

(a)

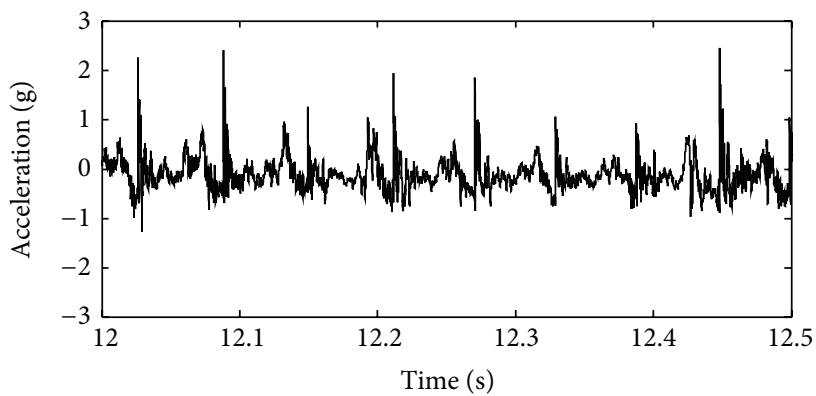

(b)

Figure 3: Vertical accelerations of the vehicle with built-in mecanum wheels (a) at low speed and (b) at high speed.

than those along the negative (downward) direction. It is noteworthy that this asymmetrical phenomenon is move evident at high- rather than low-driving speeds. In other words, the peak amplitudes of the positive accelerations are much higher than those of negative accelerations, as shown in Figure 3(b). At high speeds, the magnitudes of positive accelerations are nearly three times greater than those of negative accelerations. It is supposed that the asymmetrical behavior is caused by the structural shape of the mecanum wheel on which the rollers are inclined by $45^{\circ}$ with respect to each other. Therefore, in order to investigate the asymmetrical phenomenon in detail, a quantitative analysis of the dynamic characteristics is performed, and the contact mechanism of the mecanum wheel is explained in the next section.

\section{Dynamic Simulation of the Mecanum Wheel}

In this chapter, dynamic simulations of a vehicle with builtin mecanum wheels were carried out by using RecurDyn [23] software to investigate the asymmetric behaviors of accelerations. The roller slip of the mecanum wheel was also considered to replicate the actual driving environment. In addition, the peak to peak and the RMS values of the displacements and the accelerations of the vehicle with built-in mecanum wheels were analyzed, and the optimal range of the curvature minimizing the vertical vibration was proposed.

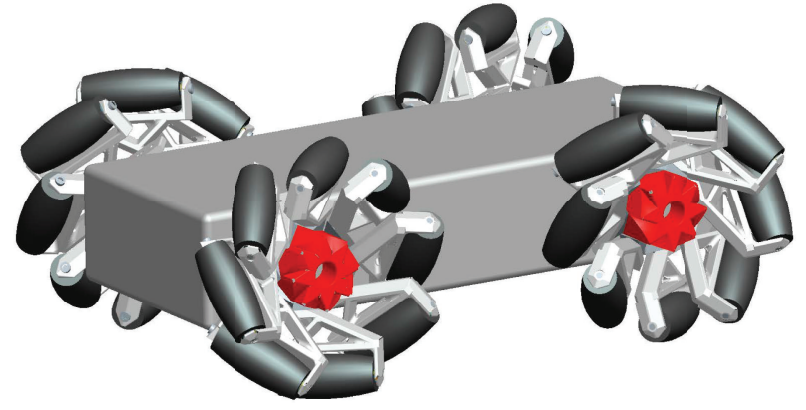

FIGURE 4: Dynamic simulation model for a proposed mecanum wheel.

3.1. Characteristics of the Vertical Accelerations of the Wheel and Vehicle Body. The vehicle model consists of the vehicle body and four mecanum wheels, as shown in Figure 4. The vehicle body, which is a cuboid shape, was used in order to avoid the asymmetric effect due to the body shape. The height, width, and length of the vehicle body were $150 \mathrm{~mm}$, $300 \mathrm{~mm}$, and $750 \mathrm{~mm}$, respectively. Four mecanum wheels and the body were connected with revolute joints and the driving torque was applied to each revolute joint. The mass of the vehicle body and the mecanum wheels were $266 \mathrm{~kg}$ and $14 \mathrm{~kg}$, respectively. Also, the static and dynamic friction coefficients between the rollers and ground were set as 0.5 and 


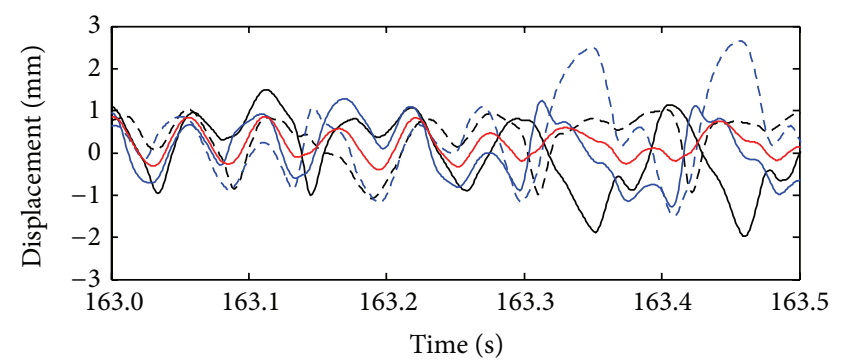

(a)

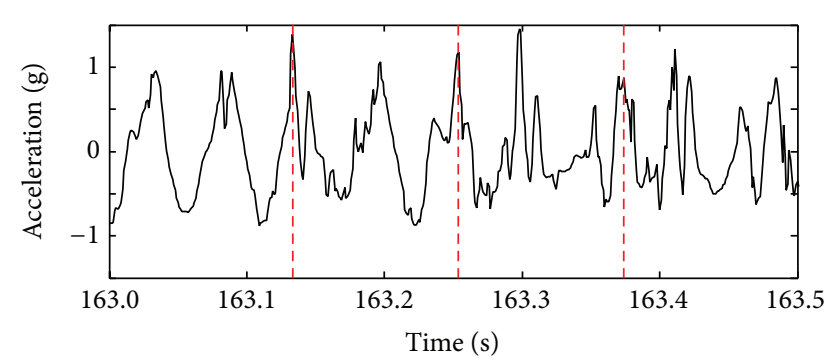

(b)

FIGURE 5: Simulation results of the vehicle and mecanum wheels. (a) Vertical displacements of the vehicle body (red solid line) and each wheel (blue dash: left front wheel, blue solid: right front wheel, black dash: left rear wheel, and black solid: right rear wheel), (b) vertical accelerations of the vehicle body (vertical red dash line indicates the time when the changeover of rollers occurs).

0.4 , respectively. Total simulation time was 200 seconds and the sampling frequency was $1000 \mathrm{~Hz}$.

Figure 5(a) illustrates the vertical displacements of each wheel and the vehicle body, and Figure 5(b) illustrates the corresponding vertical acceleration of the vehicle body to show the phase differences. Those were calculated at the center of gravity in the average driving speed of approximately $0.75 \mathrm{~m} / \mathrm{s}$. It can be observed that each wheel oscillates randomly with respect to the time, and the vehicle body vibrates by the interaction of each wheel motion. In particular, the phase changes of each wheel were randomly repeated in the entire simulation period; for example, the phases of four wheels are quite identical initially and quickly randomized in the considered period. These phase changes can be explained by the fact that the slips between rollers and ground lead to the different angular displacement of each wheel despite applying constant torque. Moreover, the vertical displacement of the vehicle body is equal to the average of that of each wheel, because it was calculated at the geometric center of the vehicle body. Therefore it can be concluded that the combination of the slip motion and the vertical displacement of each wheel generates the vertical dynamic characteristics of the vehicle body.

The asymmetrical acceleration of the vehicle body was also observed in this simulation, as shown in Figure 5(b). The vertical red dash line indicates the time at which the roller of the left rear wheel contacted with the ground changes over. As can be seen, the peaks of the positive accelerations are higher and sharper than those of the negative accelerations. In particular, it appears that the impact force due to the contact between the rollers and road generates the peak of the vertical acceleration of the vehicle body. In other words, the vehicle body is vibrated by the sequential contacts between the rollers and the road. Finally, we considered that this type of impact mechanism due to the contact between the rollers and road mainly attributes to the asymmetric accelerations of the vehicle body with built-in mecanum wheels, and it is supposed that the vertical vibration of the vehicle body can be reduced by the geometry change of the rollers.

3.2. The Effect of the Roller Curvature to Vertical Vibration. Although the peak of the vertical acceleration is unavoidable in the mecanum wheel platform, it is possible to minimize
TABLE 1: Curvature and height of the nine rollers used in the computations.

\begin{tabular}{lcc}
\hline Model & Curvature $\left(\mathrm{mm}^{-1}\right)$ & $H_{\text {roller }}(\mathrm{mm})$ \\
\hline 1 & 0.0011 & 26 \\
2 & 0.0026 & 23 \\
3 & 0.0037 & 21 \\
4 & 0.0042 & 20 \\
5 & 0.0047 & 19 \\
6 & 0.0052 & 18 \\
7 & 0.0056 & 17 \\
8 & 0.0066 & 15 \\
9 & 0.0075 & 13 \\
\hline
\end{tabular}

the vibrations of the mecanum wheel choosing the optimal parameter of the rollers. To do this, we calculated the vertical displacements and accelerations of the vehicle body with respect to the several curvatures of the roller shown in Figure 6. The displacements of the wheel depend on the distance between the center of the wheel and the contact point of the roller with the road, and thus we expected that the roller curvature has the optimal values to minimize the vertical vibration. Therefore the curvature of the rollers was set as the design parameter, and the nine curvatures were analyzed in this study. The geometric data of the rollers are listed in Table 1. Geometrically the curvature of a straight line is defined to zero. Also in order to investigate the effects of the speed, the low, mid, and high speeds of the vehicle were taken into account setting approximately $0.5 \mathrm{~m} / \mathrm{s}, 0.75 \mathrm{~m} / \mathrm{s}$, and $1.0 \mathrm{~m} / \mathrm{s}$, respectively. The vertical displacement and acceleration were extracted at the center of gravity of the vehicle body. Then, the peak to peak and root mean square (RMS) values of displacement and accelerations were calculated with respect to the roller curvatures. In order to minimize the transient effects of the vehicle motion, the peak to peak and RMS values were computed using the data during the time between 30 and 200 seconds.

Figure 7 shows the peak to peak and RMS values of displacements and accelerations with respect to the roller curvatures and the driving speeds. Comparing Figures 7(a) and $7(\mathrm{~b})$, the speed dependency is clear in the acceleration 


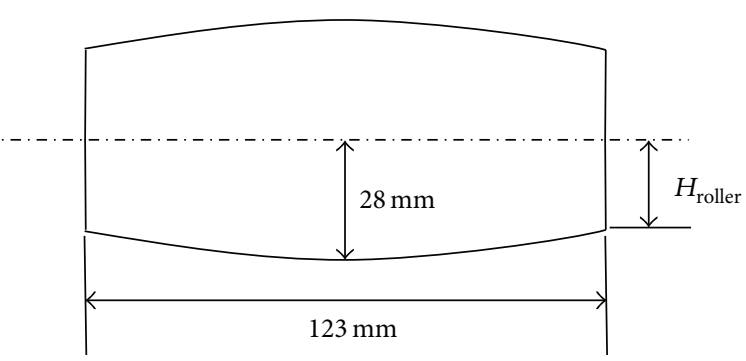

(a) Geometry of the rollers
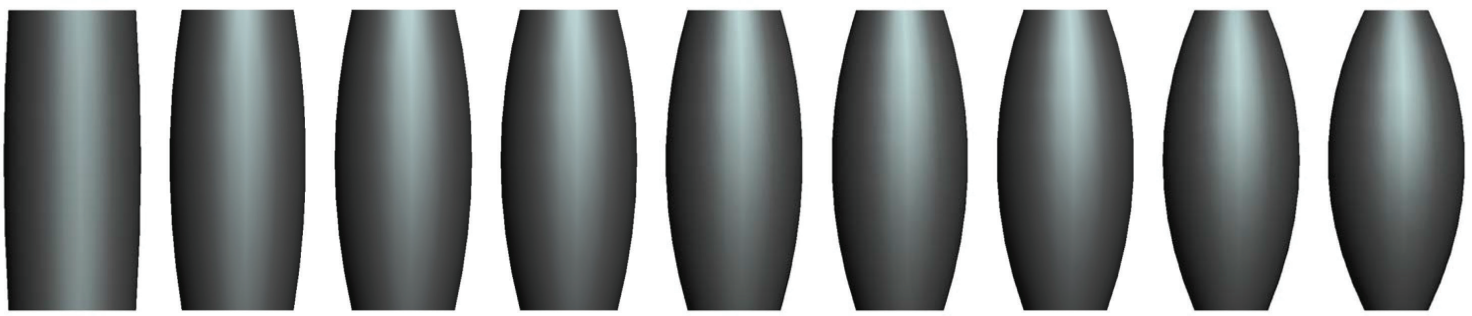

(b) Nine rollers of different curvatures used in the dynamic simulations

FIGURE 6: The geometry and shape of the rollers.
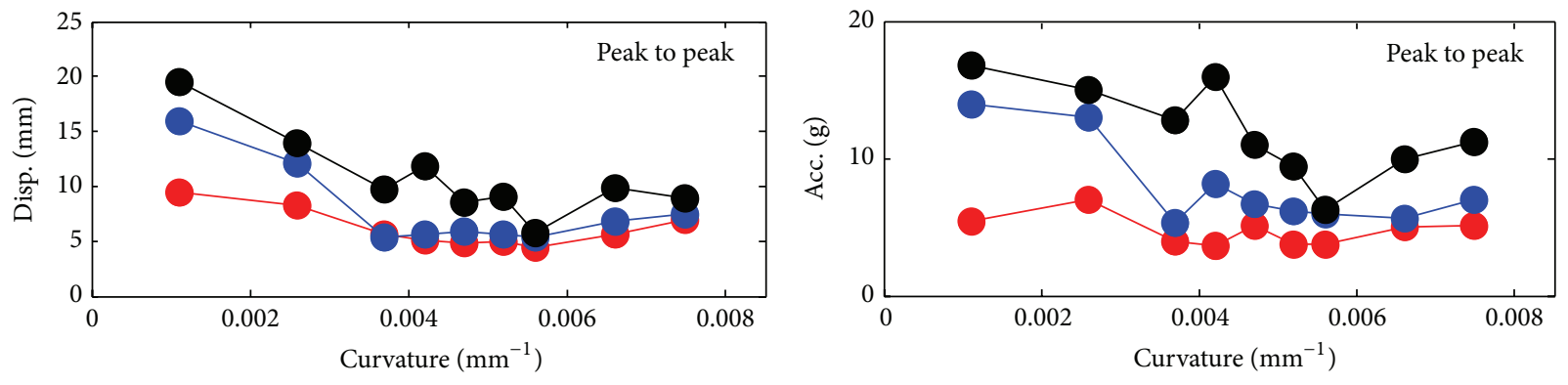

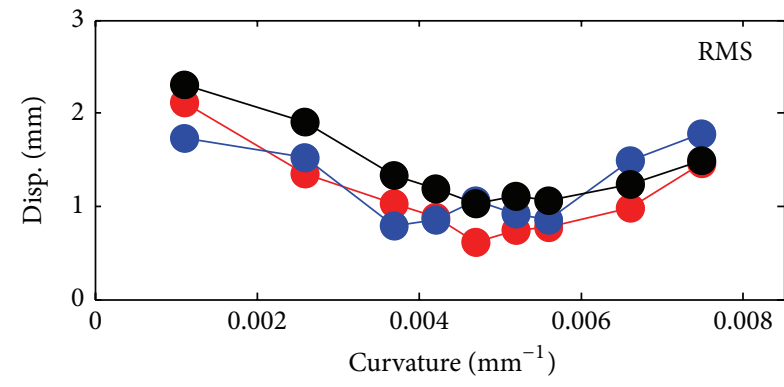

(a) Displacement

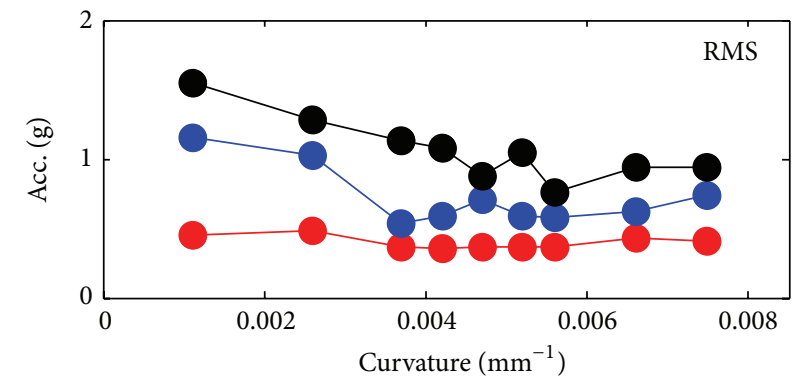

(b) Acceleration

FIGURE 7: Simulation results of the vehicle vibrations with respect to nine curvatures of rollers and three driving speeds of the vehicle. (a) Displacement and (b) acceleration (red, blue, and black lines indicate low, mid, and high speed, resp., and top and bottom figures indicate peak to peak and RMS values, resp.).

results, even though the calculation values of the mid speed are not equal to the average speed of the low and high speeds. However, the curvature dependency is obvious in the displacement results. In particular, the RMS values of the displacements show the same tendency regardless of the driving speeds. We reasoned that the displacements of the vehicle body are dominated by the geometry of the roller. In addition, the peak to peak results show the more complicated pattern than the RMS results with respect to the curvature. Also those variations of the accelerations were increased with the increasing speed because of the inertia effect. For example, the peak to peak value of the acceleration at 1st curvature is similar with that of 7 th curvature in the low speed; however the difference of them occurs approximately three times in the high speed.

The calculation values of the displacements and accelerations tend to increase with the sharp or excessively rounded edge of the roller. This pattern is also more distinct in the displacement results than the accelerations. It might seem reasonable that the excessive curvature results in the increased 


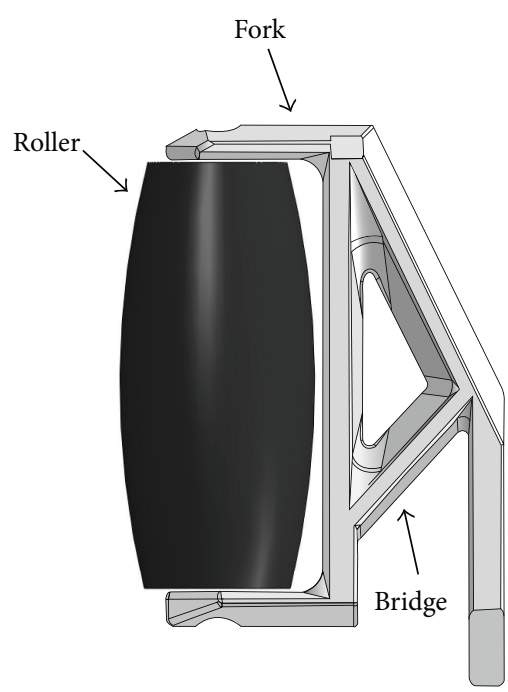

(a) Original design

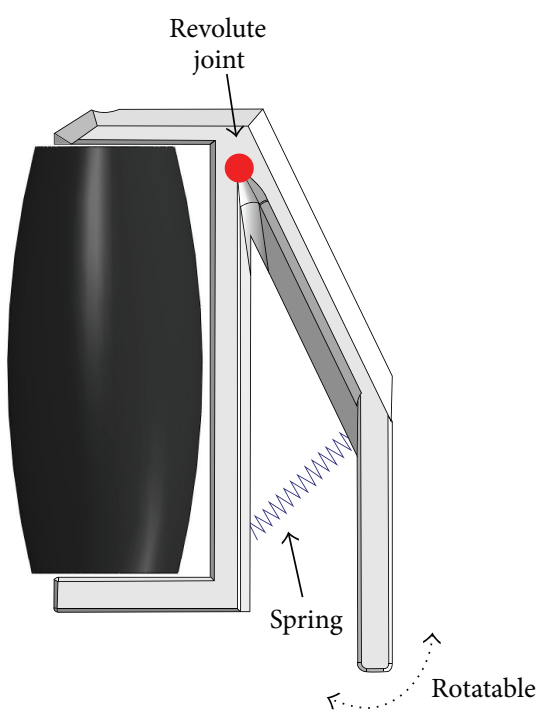

(b) Proposed design

FIgURE 8: Original and a proposed design with a spring and a revolute joint.

displacements and the accelerations due to the unintended bumpy motion of the vehicle when the changeover of the rollers occurs. On the other hand, adequate curvature leads to the lower displacements and the accelerations because the contact point of the roller is smoothly changed to the next roller. Indeed, the local minimum values were observed clearly in the RMS of the displacements. And those were also weakly shown in the acceleration results. In this study, we considered that the optimal curvature is in the range between 3rd and 7th curvatures.

\section{Design Change of the Fork}

In this chapter, the alternative design of the fork was proposed in order to additionally reduce the vertical vibration of the vehicle body. The spring to absorb the impact force caused by the contact of the rollers was used to suppress the vertical vibration, and the dynamic simulation of the proposed design was performed. In addition, the equivalent stiffness of the fork was calculated using the finite element analysis.

4.1. Spring to Attenuate the Impact Force. To suppress the vertical vibration of the mecanum wheel, the design of the fork was modified using the spring, as shown in Figure 8. The bridge was replaced with a spring and a frictionless revolute joint in the proposed design. The spring can be expected to attenuate the vibration and shock caused by the contact of the rollers and the inertia effect of the vehicle body. The revolute joint is used to allow the stretch and compression of the spring, because the dynamic simulation generally considers rigid body motion; the spring cannot be deformed without the rotation of the fork. Now, we carried out the parametric study of the proposed design in order to determine the optimal value of the spring stiffness. Similar with the previous section, the peak to peak and RMS values of the vertical displacement and acceleration of the vehicle body were calculated with respect to the spring stiffness. The fourth curvature, as an example of the roller curvature, was chosen in this simulation at the low, mid, and high driving speeds. The calculations were performed for the seven values of stiffness in the range between $100 \mathrm{~N} / \mathrm{mm}$ and $400 \mathrm{~N} / \mathrm{mm}$.

Figure 9 shows the peak to peak values and RMS values of the displacement and the acceleration at the CG point of the vehicle body. It is noted that the vertical accelerations are significantly reduced by the shock absorption of the spring. Compared to absence of the spring, the peak to peak values of the acceleration in the presence of $200 \mathrm{~N} / \mathrm{mm}$ spring were reduced with $64.4 \%, 83.0 \%$, and $81.4 \%$, and the RMS values of acceleration were reduced with $60.5 \%, 75.0 \%$, and $71.5 \%$ at the low, mid, and high speeds, respectively. On the other hand, the RMS values of the displacements slightly decreased in the presence of the spring because they are mainly determined by the geometry of the rollers.

From Figure 9, the curves of the displacement and acceleration values are smoother than the results with respect to the curvatures shown in Figure 7. It could be inferred that the contact forces of the rollers are uniformly generated by the shock-absorbing effect of the spring. Also, the speed dependency was clearer below the stiffness of $200 \mathrm{~N} / \mathrm{mm}$. Because it is obvious that the lower stiffness leads to the larger deflection of the spring, it causes higher vertical displacement and acceleration of the vehicle body.

In addition, the local minimum values were consistently found in all results with respect to the spring coefficient and the driving speed. Because the lower stiffness is insufficient to resist the vertical force, it leads to the higher displacements and the accelerations. Furthermore, all result values are increased with the increasing of the stiffness. The previous results with 4 th curvature can be reasonably assumed the result values with the infinite stiffness of the spring. Therefore, the displacement and the acceleration values with respect to the stiffness could ultimately converge to the infinite stiffness 

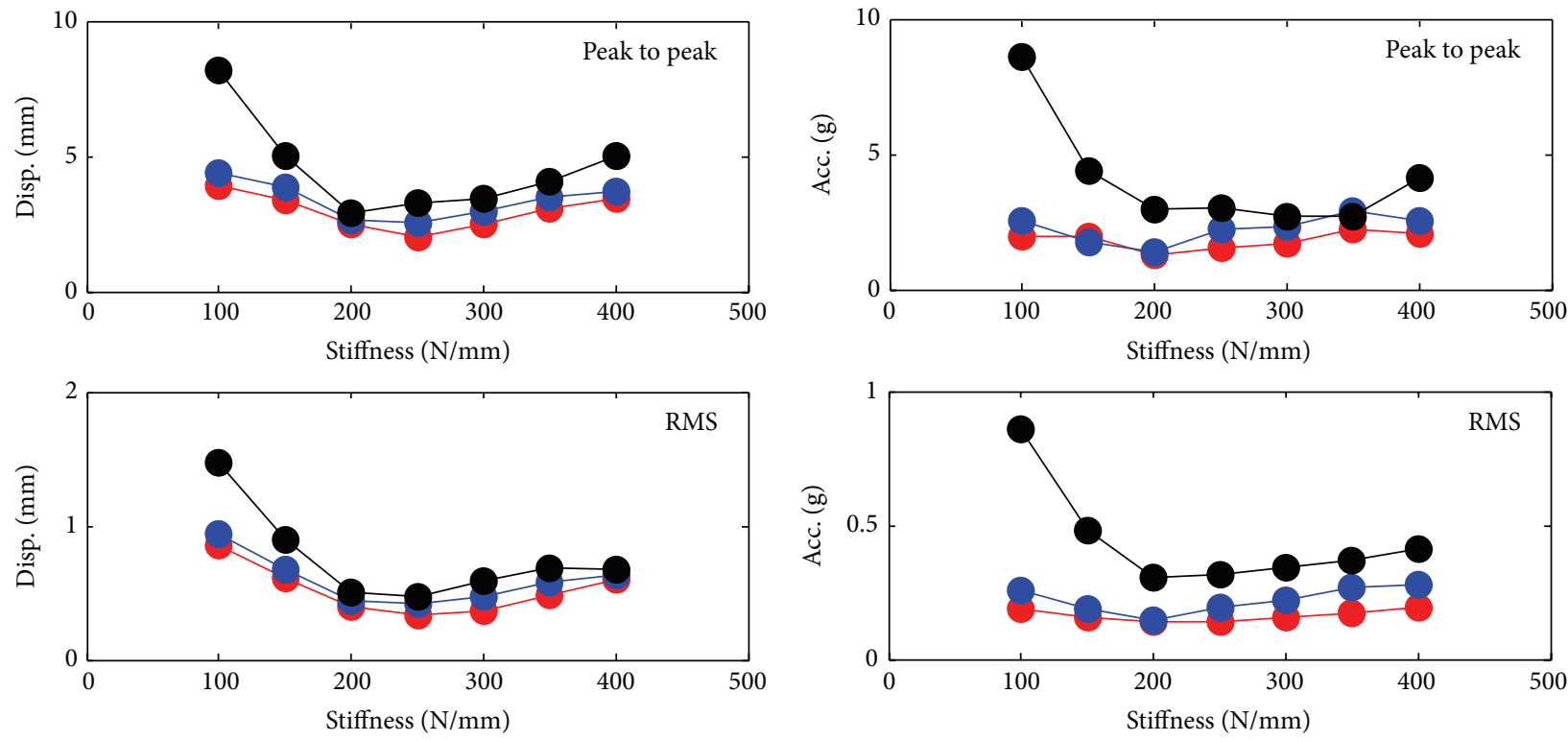

(a) Displacement

(b) Acceleration

FIGURE 9: Simulation results of the vehicle vibrations with respect to eight spring stiffness values and three driving speeds of the vehicle. (a) Displacement and (b) acceleration (red, blue, and black lines indicate low, mid, and high speed, resp., and top and bottom figures indicate peak to peak and RMS values, resp.).

results. Actually, it is observed that the calculation values are gradually increased with the increasing the stiffness. In other words, the displacement and acceleration results are increased with the lower or the higher stiffness, and it is essential that the results with respect to the stiffness have the local minimum. In this study, the optimal spring stiffness was determined at the range between 200 and $250 \mathrm{~N} / \mathrm{mm}$.

4.2. Equivalent Stiffness of the Fork. We calculated the equivalent stiffness of the fork based on the dynamic simulation results. For vertical vibration, the bending of the fork was considered instead of the spring. The analysis of the bending behavior was carried out using the finite element method software, Abaqus/Standard [24], because the dynamic simulation in this study was performed using the rigid body analysis. The vertical force applied to the fork, and the deflection was extracted at the tip of the fork in order to calculate the equivalent stiffness from the slope of the forcedeflection relationship. The force and boundary conditions are shown in Figure 10. In addition, the radius of the fillet was set as the design parameter to extract the optimal stiffness. The radius of the fillet means the radius of a circle rounding the corner of the fork, as illustrated by Figure 10. In this analysis, the corner of the fork becomes thicker as the radius of the fillet increases; in other words, the fork is getting stiffer. The material of the fork was aluminum with Young's modulus of $70 \mathrm{GPa}$, Poisson's ratio of 0.33 . Also, the vertical force was applied with range of 0 to $1000 \mathrm{~N}$. The fillet radii and the corresponding stiffness of the fork are listed in Table 2. The equivalent stiffness of the fork was proportionally increased with the increase of the radius of the fillet. Also the optimal spring value of $250 \mathrm{~N} / \mathrm{mm}$ was shown in between the fillet radii of 2 and $3 \mathrm{~mm}$.

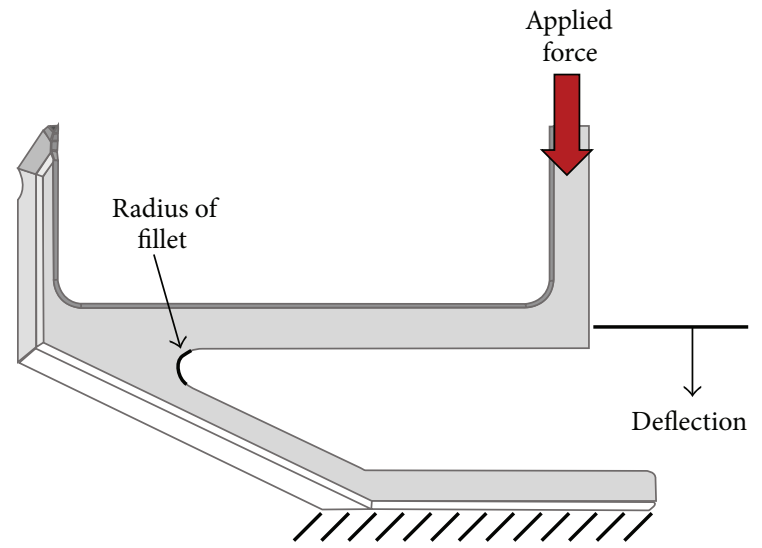

Fixed surface

FIGURE 10: Force and the boundary conditions and the design parameter of the fork.

TABLE 2: Equivalent stiffness with respect to the radius of fillet.

\begin{tabular}{lc}
\hline Radius of fillet $(\mathrm{mm})$ & Stiffness $(\mathrm{N} / \mathrm{mm})$ \\
\hline 0 & 201.21 \\
1 & 222.86 \\
2 & 245.82 \\
3 & 272.67 \\
4 & 303.23 \\
5 & 339.37 \\
6 & 382.49 \\
\hline
\end{tabular}

\section{Conclusions and Discussions}

In this study, we performed the quantitative analysis of the mecanum wheel vibration and proposed the mecanum wheel 
to suppress the vertical vibration. A mecanum wheel has the inevitable vibration due to the repeated contact of the rollers. However, the quantitative analysis of the vibration is not actively researched. For quantitative analysis, we made the prototype of mecanum wheel and measured those vertical vibrations. As a result, the asymmetric acceleration was observed in the experimental results. The dynamic simulation was also performed in order to investigate the vertical vibration characteristic of the vehicle body with built-in mecanum wheels. In addition, we proposed the optimal roller curvature to minimize the vertical vibration based on the peak to peak and RMS values of the displacements and accelerations. To additionally reduce the vertical vibration, we suggested the modified design of the fork, and the vertical accelerations were dramatically decreased. Finally, the equivalent stiffness of the fork was calculated using the finite element analysis.

\section{Conflict of Interests}

The authors declare that there is no conflict of interests regarding the publication of this paper.

\section{Acknowledgment}

This research was supported by the MSIP (Ministry of Science, ICT and Future Planning), Korea, under the C-ITRC (Convergence Information Technology Research Center) (IITP-2015-H8601-15-1002) supervised by the IITP (Institute for Information \& Communications Technology Promotion).

\section{References}

[1] G. Campion, G. Bastin, and B. D’Andréa-Novel, "Structural properties and classification of kinematic and dynamic models of wheeled mobile robots," IEEE Transactions on Robotics and Automation, vol. 12, no. 1, pp. 47-62, 1996.

[2] M. Wada and S. Mori, "Holonomic and omnidirectional vehicle with conventional tires," in Proceedings of the 13th IEEE International Conference on Robotics and Automation, pp. 3671-3676, IEEE, Minneapolis, Minn, USA, April 1996.

[3] R. Holmberg and O. Khatib, "Development and control of a holonomic mobile robot for mobile manipulation tasks," International Journal of Robotics Research, vol. 19, no. 11, pp. 1066-1074, 2000.

[4] H. Yu, M. Spenko, and S. Dubowsky, "Omni-directional mobility using active split offset castors," Journal of Mechanical Design, vol. 126, no. 5, pp. 822-829, 2004.

[5] F. G. Pin and S. M. Killough, "A new family of omnidirectional and holonomic wheeled platforms for mobile robots," IEEE Transactions on Robotics and Automation, vol. 10, no. 4, pp. 480489, 1994.

[6] C. Leng, Q. Cao, and Y. Huang, "A motion planning method for omni-directional mobile robot based on the anisotropic characteristics," International Journal of Advanced Robotic Systems, vol. 5, no. 4, pp. 327-340, 2008.

[7] H. Rajaie, O. Zweigle, K. Häussermann, U.-P. Käppeler, A. Tamke, and P. Levi, "Hardware design and distributed embedded control architecture of a mobile soccer robot," Mechatronics, vol. 21, no. 2, pp. 455-468, 2011.
[8] J. De Best, R. Van de Molengraft, and M. Steinbuch, "A novel ball handling mechanism for the RoboCup middle size league," Mechatronics, vol. 21, no. 2, pp. 469-478, 2011.

[9] B. E. Ilon, "Wheels for a course stable selfpropelling vehicle movable in any desired direction on the ground or some other base," US Patent No. 3,876,255, 1975.

[10] J. Kim, S. Woo, J. Kim, J. Do, S. Kim, and S. Bae, "Inertial navigation system for an automatic guided vehicle with mecanum wheels," International Journal of Precision Engineering and Manufacturing, vol. 13, no. 3, pp. 379-386, 2012.

[11] J. W. Kang, H. S. Hong, B. S. Kim, and M. J. Chung, "Assistive mobile robot systems helping the disabled workers in a factory environment ", International Journal of Assistive Robotics and Mechatronics, vol. 9, no. 2, pp. 42-52, 2008.

[12] S. W. Yoon, S. Park, and J. S. Kim, "Kalman filter sensor Fusion for mecanum wheeled automated guided vehicle localization," Journal of Sensors, vol. 2015, Article ID 347379, 7 pages, 2015.

[13] J. A. Cooney, W. L. Xu, and G. Bright, "Visual dead-reckoning for motion control of a mecanum-wheeled mobile robot," Mechatronics, vol. 14, no. 6, pp. 623-637, 2004.

[14] S. L. Dickerson and B. D. Lapin, "Control of an omni-directional robotic vehicle with Mecanum wheels," in Proceedings of the IEEE National Telesystems Conference (NTC '91), vol. 1, pp. 323328, March 1991.

[15] A. Gfrerrer, "Geometry and kinematics of the mecanum wheel," Computer Aided Geometric Design, vol. 25, no. 9, pp. 784-791, 2008.

[16] L. Ferrière, B. Raucent, and G. Campion, "Design of omnimobile robot wheels," in Proceedings of the IEEE International Conference Proceedings on Robotics and Automation, vol. 4, pp. 3664-3670, Minneapolis, Minn, USA, April 1996.

[17] K.-S. Byun and J.-B. Song, "Design and construction of continuous alternate wheels for an omnidirectional mobile robot," Journal of Robotic Systems, vol. 20, no. 9, pp. 569-579, 2003.

[18] J.-B. Song and K.-S. Byun, "Design and control of a fourwheeled omnidirectional mobile robot with steerable omnidirectional wheels," Journal of Robotic Systems, vol. 21, no. 4, pp. 193-208, 2004.

[19] J.-B. Song and K.-S. Byun, "Steering control algorithm for efficient drive of a mobile robot with steerable omni-directional wheels," Journal of Mechanical Science and Technology, vol. 23, no. 10, pp. 2747-2756, 2009.

[20] A. Weiss, R. G. Langlois, and M. J. D. Hayes, "Dynamics and vibration analysis of the interface between a non-rigid sphere and omnidirectional wheel actuators," Robotica, vol. 33, no. 9, pp. 1850-1868, 2014.

[21] B. Dehez, V. Froidmont, D. Grenier, and B. Raucent, "Design, modeling and first experimentation of a two-degree-offreedom spherical actuator," Robotics and Computer-Integrated Manufacturing, vol. 21, no. 3, pp. 197-204, 2005.

[22] M. Wada and H. H. Asada, "Design and control of a variable footprint mechanism for holonomic omnidirectional vehicles and its application to wheelchairs," IEEE Transactions on Robotics and Automation, vol. 15, no. 6, pp. 978-989, 1999.

[23] FunctionBay Inc, Recurdyn/Solver Theoretical Manual, 2012.

[24] Dassault Systems, Abaqus 6.13 Analysis User Manual, 2013. 


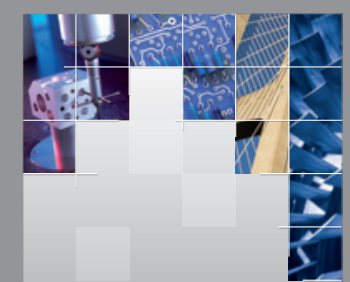

\section{Enfincering}
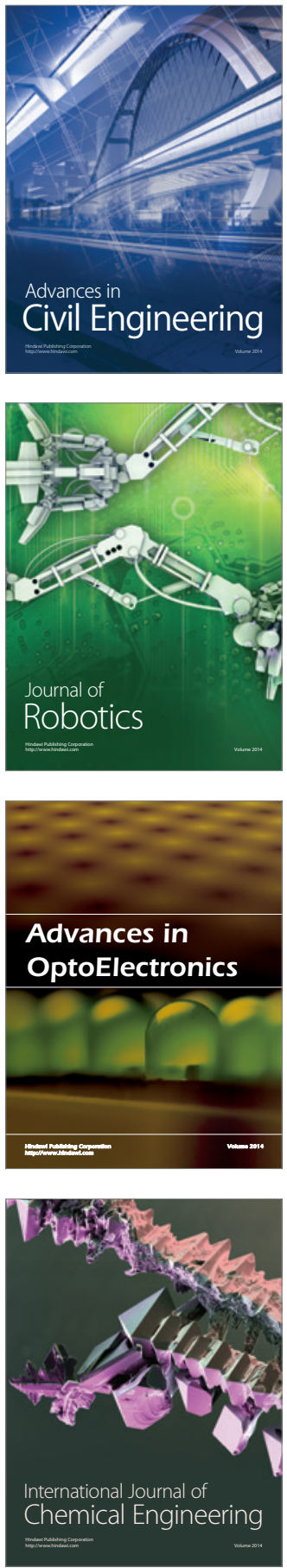

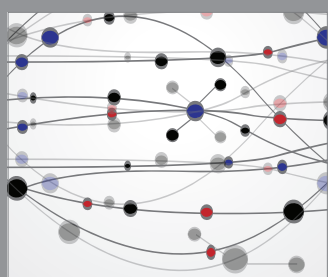

The Scientific World Journal

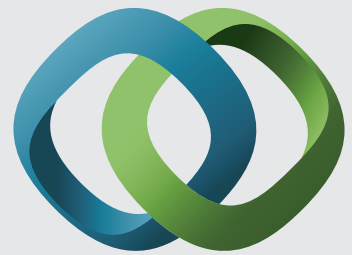

\section{Hindawi}

Submit your manuscripts at

http://www.hindawi.com
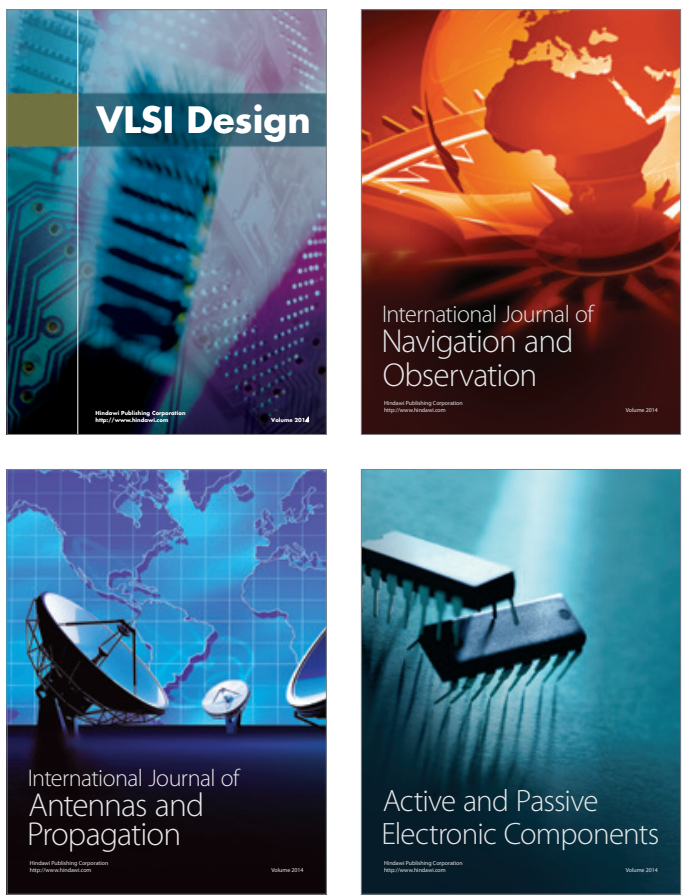
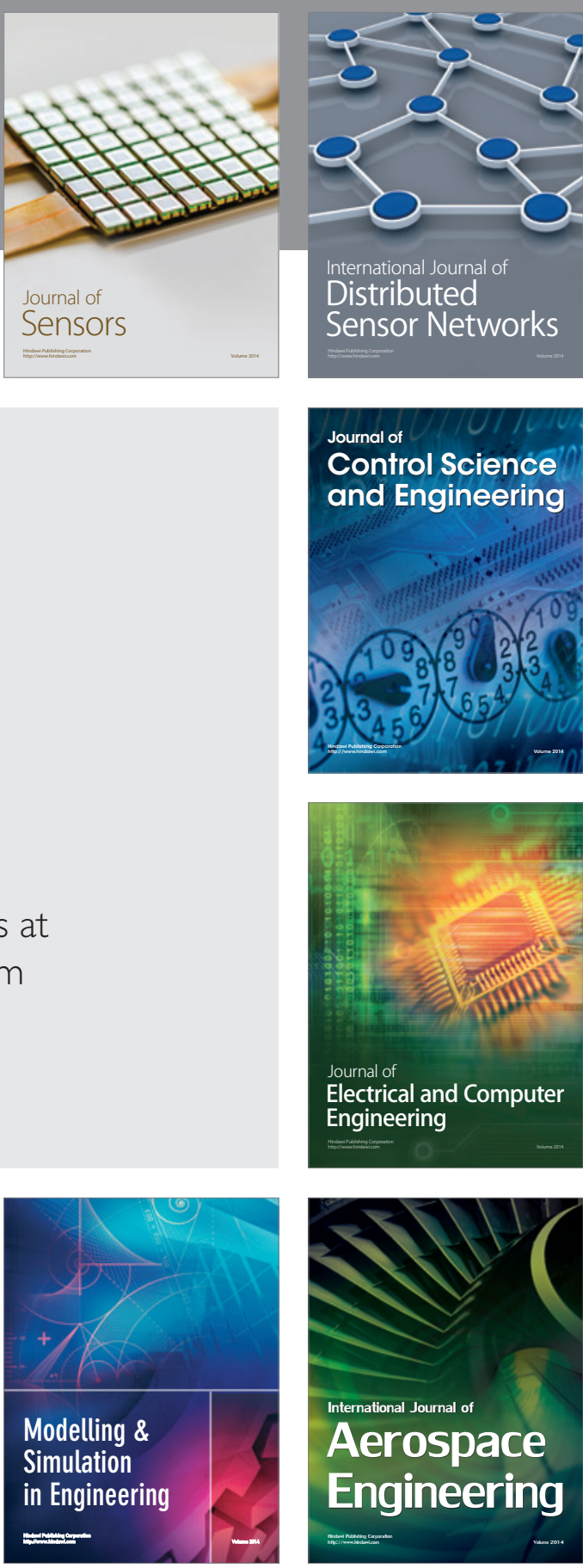

International Journal of

Distributed

Sensor Networks

Journal of

Control Science

and Engineering
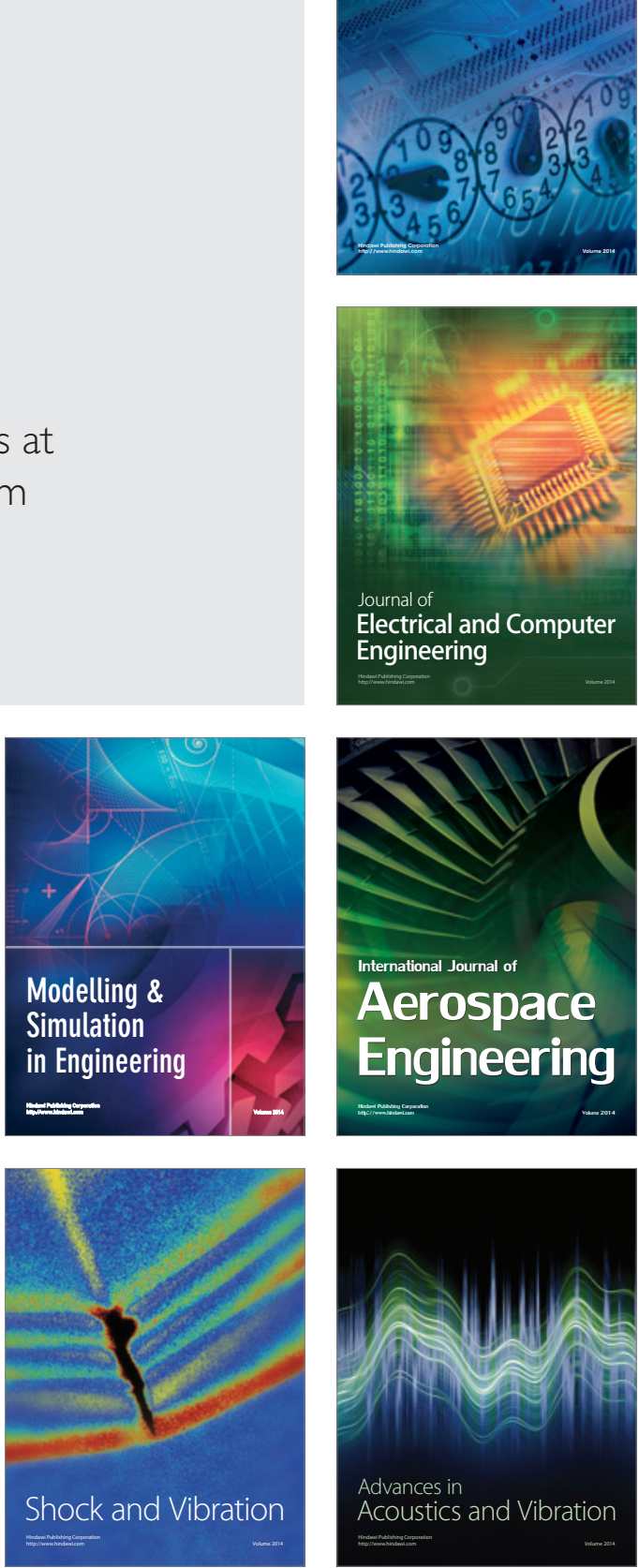